\title{
FROM POINT CLOUD TO DIGITAL FABRICATION: A TANGIBLE RECONSTRUCTION OF CA' VENIER DEI LEONI, THE GUGGENHEIM MUSEUM IN VENICE
}

\author{
C. Balletti *, F. D’Agnano, F. Guerra, P. Vernier \\ Università Iuav di Venezia \\ balletti@iuav.it, (fdagnano,guerra2, vernier)@iuav.it
}

Commission V, WG V/2

KEY WORDS: Cultural Heritage, Laser scanning, SfM, Digital reconstruction, Digital fabrication

\begin{abstract}
:
The paper describes how new digital methodologies can be used within the field of Cultural Heritage, not only with the aim of documenting the actual state of an architecture but to review the past transformations it has undergone, conserving and representing these histories as well. The premise to conservation and enhancement of our Heritage is a deep study in terms of position, shape, colour, and also of the historical and artistic features.

Survey methods have acquired data acquisition techniques in line with technological progress: today's electronic and IT technologies, that are the tools of modern Geomatics, allow the effective survey and representation of 3D objects, from architectural structures to sculptures or archaeological finds.

Over the last few years, the methodologies of acquisition and integrated representation for 3D patrimony documentation have developed and consolidated considerably: the possibilities of the digital realm can augment the understanding and the valorisation of a monument.

The specific case offered in the present paper, Ca' Venier dei Leoni, the palace where is the Guggenheim Museum in Venice, is a significant example. It suggests not only the theme of the "no longer existing", or better never built, but also the opportunity to formulate hypotheses regarding its implementation and the impact that the palace would have had in the Venetian contest
\end{abstract}

\section{INTRODUCTION}

Geomatics, within the field of Cultural Heritage, has the role of identifying tools and methods in surveying activities, data processing and representation models as well as in data recording and dissemination. Particularly, considering documentation for study, knowledge, conservation and renovation applications (Balletti et al. 2015; Bitelli, Mannina 2010), geomatic techniques allow a metric and qualitative description that can make use of different study and survey methods that can interface thanks to the availability of the methods and algorithms of data transformation and merging. Methods and tools used in the survey of Cultural Heritage (CH) cover a wide range according to the characteristics of the studied object and of the required accuracy. Surveying is a very complex operation both from the methodological and operational point of view: because it requires critical interpretation abilities for a correct comprehension of the surveyed object from which the correct strategies can be derived to obtain the best result from the survey operations (Guidi et al. 2004; Cignoni et al. 2008; Remondino 2010; Chiabrando et al. 2014; Balletti et al, 2014).

Today, the actual spectrum of methodologies and instruments is extremely wide and, often, the multi-resolution approach and the application of different techniques and methodologies provide better results in terms of accuracy and optimisation of the final product.

As can be understood from the latest research experiences (Guidi et al, 2009; Citlalli Gamez et al., 2015; Tucci, Bonora, 2015), the state of art approach for $\mathrm{CH} 3 \mathrm{~d}$ documentation uses and integrates multiple sensors and technologies (photogrammetry, laser scanning, topography, etc.) taking advantage from the intrinsic potentials of each technique, bridging over the individual weaknesses of each method alone and achieving more accurate and complete geometric surveying for interpretation, representation and digital conservation issues (Stanco et. al., 2011).

In the last few years, in the field of $\mathrm{CH}$, laser scanners (triangulation or time-of-flight) and structured-light systems were very successful, making easier the acquisition process of data related to the geometry and shape of both simple and more complex structures (Balletti et al. 2014; Andrews et al., 2013; Tucci et al. 2013; Bonfanti et al. 2013).

In general, active sensors can provide directly and in very short time a huge amount of $3 \mathrm{D}$ data that are normally returned as a dense point cloud to which can also be added the radiometric value for each single point. The relative ease of point-clouds acquisition influenced the quick spreading of this technology. At the same time, the high morphological complexity of the objects made it essential for the treatment of data, the development of original and new software different from those used in the territorial applications (Rinaudo 2011). The amount of data requires long periods of processing (registration, classification, segmentation) and editing in order to extract the most relevant geometrical elements, or to create continuous geometrical models.

Photogrammetry, that has always been the most used methodology since the first applications of Geomatics to $\mathrm{CH}$, is today a widely used technique that can be applied to object survey, architecture and land survey for documentation, control and restoration.

\footnotetext{
* Corresponding author
} 
Especially in the last few years, thanks to IT development and the spread of digital images, interesting software and hardware solutions were proposed in order to reduce the costs of instruments and to expand the pool of users that today include also non-experts without a specific training.

These instruments are widely used for 3D reconstructions of architectural parts, for the rigorous modelling of lands and cities or monuments and statues, by creating complex models. This complexity comes from both the high number of acquired and processed data and the description of the documented shapes. A common feature of the systems used today is that they can be easily carried, which means that costs can also be reduced.

Laser scanning and photogrammetry give a very similar generic product also in terms of accuracy, the point cloud, which can be complementary to the others (Bastonero et al., 2014; Wenzel et al., 2012; Remondino 2010; Bonora et al., 2005)). Their integration shows a great potential, balancing the downsides of both techniques in specific fields of application. They can be used in special applications (such as with triangulation laser tools): an example can be on one side the survey of very tiny objects and on the other the creation of 3D models that can be used in the new representation methods, such as virtual exploration and navigation paths in museums or libraries or in virtually reconstructed environments.

Although it is not completely internal to Geomatics, but more to the documentation of $\mathrm{CH}$, it is necessary to consider the mode of representation of the digital data obtained from the surveying process. In a society in which visual communication now has a fundamental role in disseminating information, the 3D model appropriately adjusted through today's technological possibilities - can provide a message that is easily perceived and extremely incisive in communicating information about Cutural Heritage.

The overlapping and the integration of Geomatics with Computer Graphics is clear: alongside the more traditional Monge projections, where vectorial restitutions are integrated with orthophotos and-rectifications, three-dimensional models, also texturized, are more often proposed and they can be visualized through animation or applications of virtual reality.

\subsection{The case study: Ca' Venier of Lions}

The Venier Palace of Lions lies in Dorsoduro, on the shore of the Great Channel. The palace is a building that was never finished, called by Venetians "the unfinished palace", started in 1749 as Lorenzo Boschetti's project. The palace remained unfinished on the ground floor, so the long and low stone façade forms a rupture in the line of palaces on the Great Channel.

The two prints (dated 1749) by engraver and architect Giorgio Fossati, based on Boschetti's design, represent the project of the Venier Palace of Lions, as is the wooden model, by the architect Domenico Rizzi (1753), appointed by the Venier family to finish their factory, preserved at the Correr Museum. The designs and the model (figure 1) show some discrepancies, and these differences were considered in the reconstructive hypothesis.

Historical research documents that on May 21st, 1755, the builders started working on the mezzanines based on the prospectus towards the Great Channel, which was later stopped due to the Venier's financial crisis, "be it noted that coffers are empty and we urge for the need of new stone to finish the project". They would then try to obtain the necessary money, only to later decree "the abandonment of the project".

Today the structure still only has one floor, of Istria stone, expanding for 44 meters. It has eight mullion windows framed by pilasters on a continuous base that flank a central gallery with Attic base semi-columns. The prospect is cut on the first half floor and therefore the architectural order of the ground floor is not completed, appearing "severed". To culminate, a plain ceiling is placed on top of it, delimited by decorations full of vegetation, closing the prospect with a smooth top, which importance is second to the historical part.

\subsection{Workflow}

Nowadays geomatic tools combined with computer graphics provide an augmented understanding of building history through the use historical documents, playing a paramount role in preserving and valorising the cultural and environmental heritage.
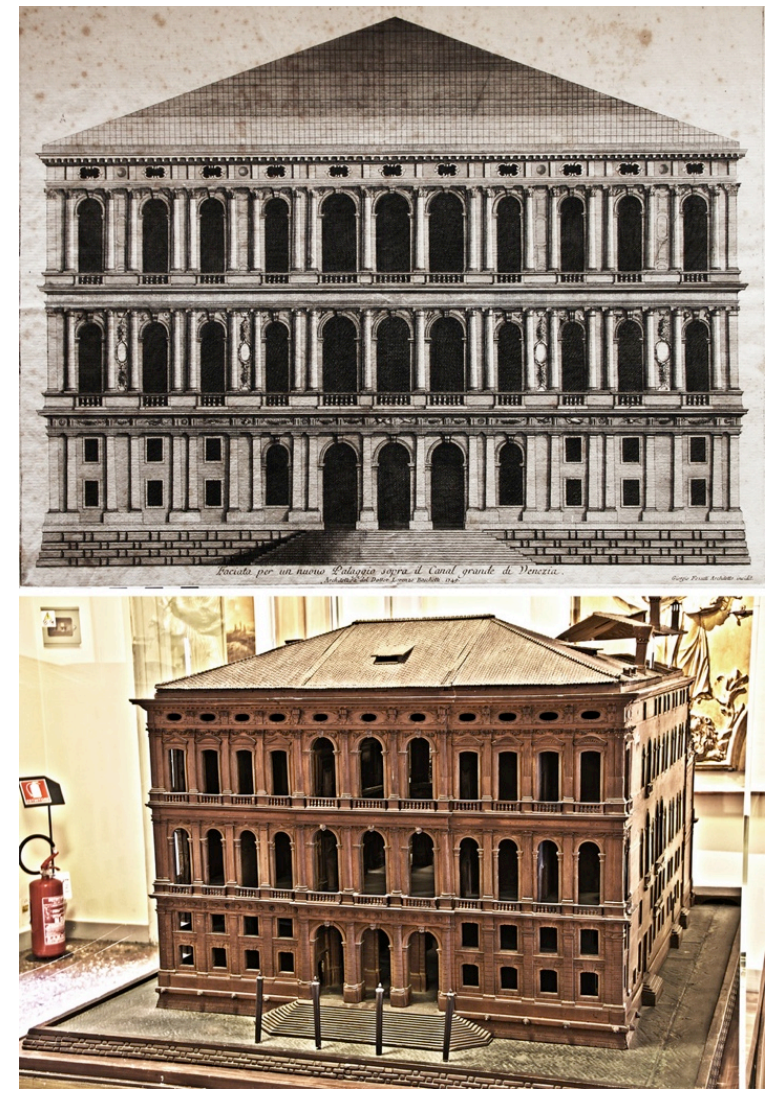

Figure 1. Comparison between Fossati's design, based on Boschetti's project, and Rizzi's wooden model

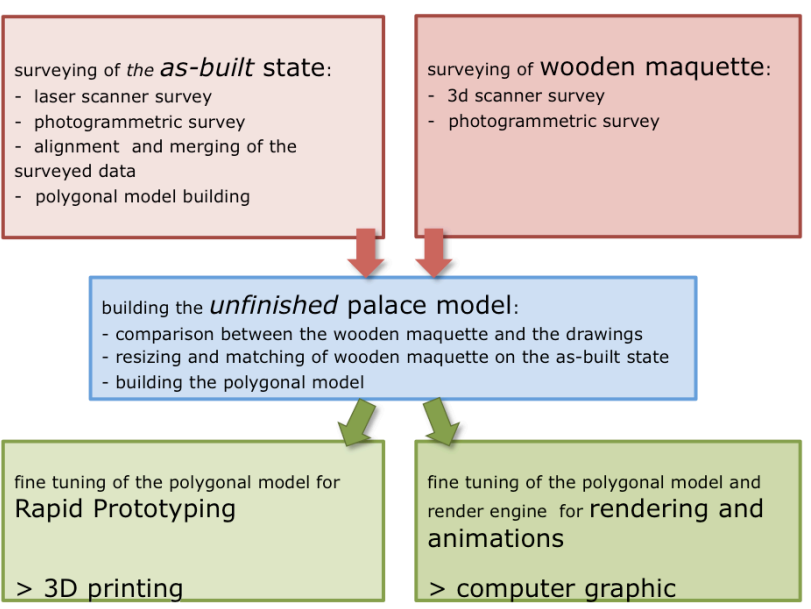

Scheme 1.Workflow 
After achieving the set goals, which were the comparison between the actual state, the historical documents that describe the projects and the virtual reconstruction of the palace, a logical plan (scheme1) was drawn up for the organization of the different phases, following a chronological order, always necessary in every phase and indispensable to move to the following ones. The work was carried out for the occasion of Ruben Camponogara's dissertation (Camponogara, 2015).

\section{SURVEYNG OF THE $A S$ BUILT STATE}

The campaign to survey the building's actual geometry was carried out with laser scanning and photogrammetric equipment. As previously stated, the procedure to integrate the photogrammetric and laser scanning data is a consolidated surveying method; it is not only used to detect geometrical figures but it also helps to improve the final radiometric data.

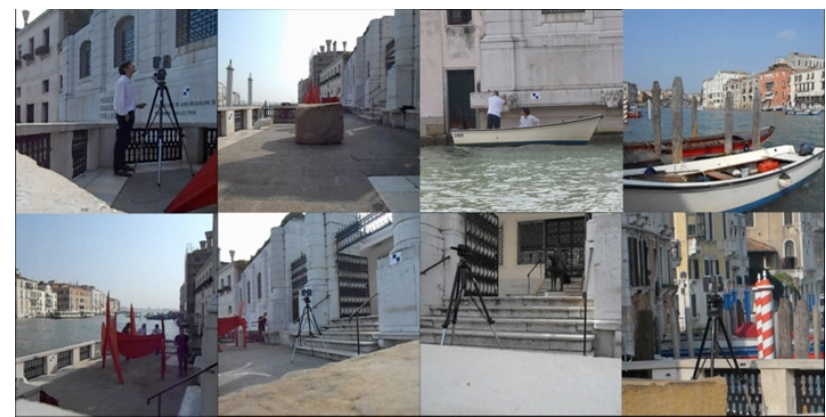

Figure 2. Phases of the laserscanning acquisition

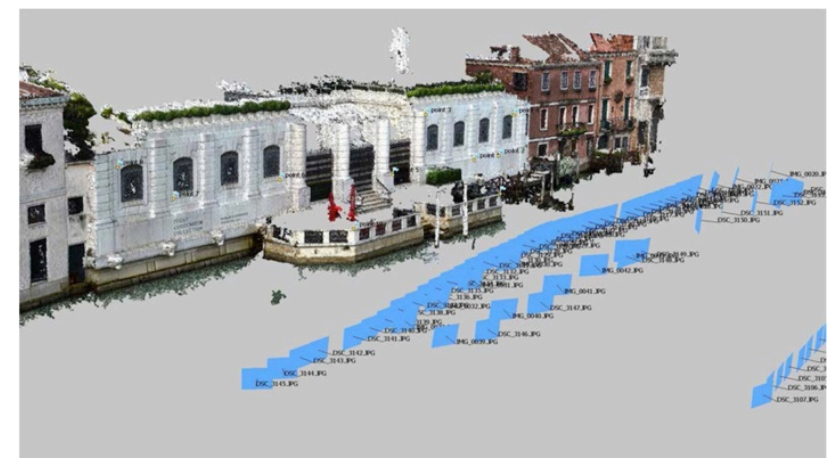

Figure 3. The SfM survey

The façade was detected with the phase shift laser scanner Focus3D by Faro (figure 2). This instrument, by now well known in the world of architectural surveying showed that, in the experiences conducted by Iuav's Photogrammetry Lab, it is particularly useful for rapid acquisitions (up to 976.000 points per second) with decent quality (with a ranging error of $\pm 2 \mathrm{~mm}$ quoted at $10 \mathrm{~m}$ and $25 \mathrm{~m}$ ) of tridimensional information, even in difficult environmental conditions.

The scans, 7 in total and taken from the terrace of the museum facing the Great Channel, and the water gate of the Corner Palace of the $\mathrm{Ca}$ ' Granda on the opposite front of the Channel, were acquired with an angular spacing of $0.035^{\circ}$, in order to guarantee the acquisition of a point each 6 to $10 \mathrm{~mm}$, in other words an adequate density for the architectural scale, for a total of 120 million points. The acquired scans were moved to the reference system using the dedicated checkboard targets, which coordinates were acquired topographically by radiation with the total station Leica TCR1103 (accuracy Hz and V: 1mgon; distance measurement accuracy: $3 \mathrm{~mm} \pm 3 \mathrm{ppm})$.
As for the photogrammetric survey, the multi-image method was preferred: by using a digital camera and software based on algorithms by Structure from Motion (SFM) and Dense Multi View 3D Reconstruction (DMVR), it is possible to estimate the parameters if interior/esxterior orientation and this way recreate the tridimensional model of the detected object (Balletti et al., 2014).

For the acquisition of the frames of the $\mathrm{Ca}$ ' Venier of Lions, a Canon EOS 550D digital camera was employed, with a focal length of $18 \mathrm{~mm}$. The camera has a CMOS APS-C 18 megapixel sensor.

The photogrammetric block was made up of two strips from two different distances (about $10 \mathrm{~m}$ and $20 \mathrm{~m}$ ), acquired from a boat along the Gran Canal. The farther set was designed for a general overview, while the one closest to acquire data with a higher resolution for the representation of the facade.

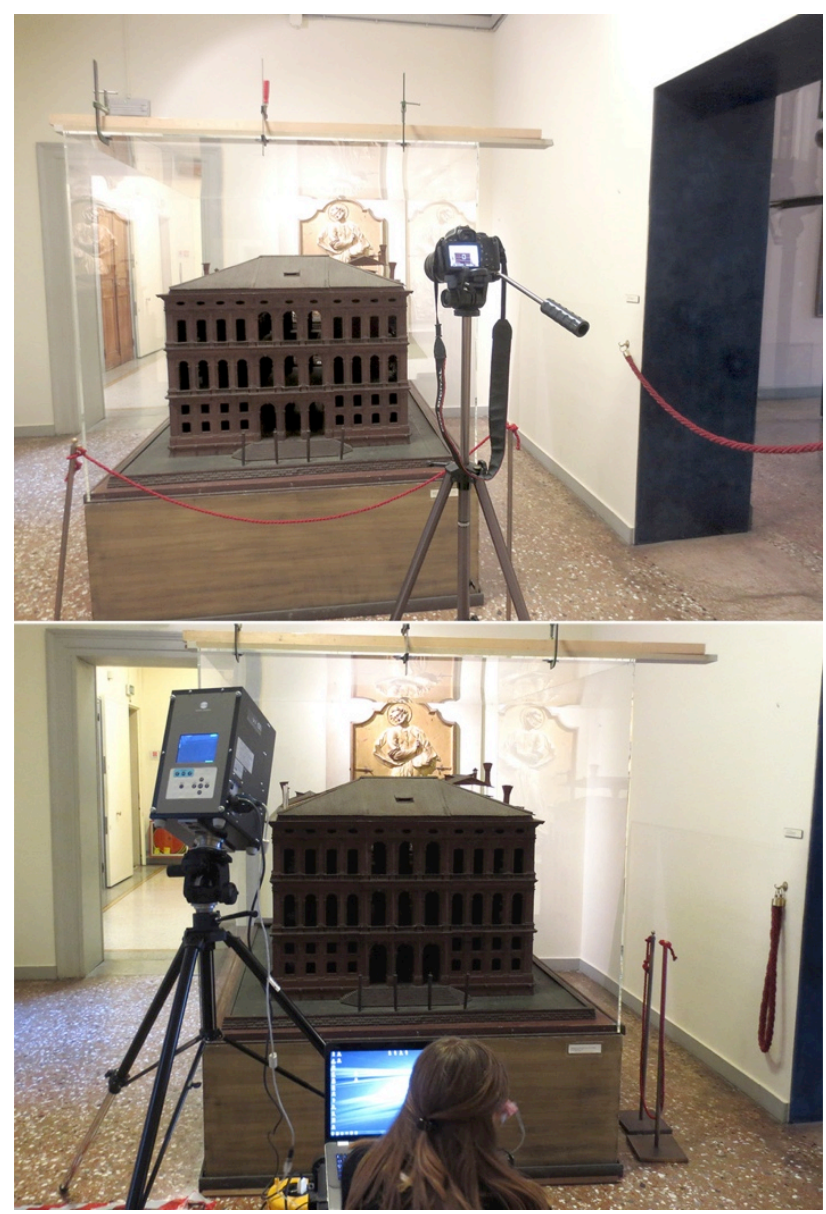

Figure 4. Photogrammetrical and laserscanning acquisition of the wooden model

A second photographic block (from a distance of $1 \mathrm{~m}$, with convergent geometry) was carried out with a Canon Ixus 125 HS $(24 \mathrm{~mm})$ for a better acquisition of the lionheads on the base. The images were processed with one of the main commercial software by Structure from Motion: Agisoft Photoscan (figure 3 ). No pre-calibration was performed, but just a self-calibration executed during the bundle-block adjustment itself. Thanks to a matching algorithm of corresponding points on the various images, the position of the camera and the scene's geometry was pieced together; a sparse point cloud could then be visualized, made up of the points used in the matching phase. For the phase of absolute orientation, to georeferenced the 
models in the surveyed reference system, some architectural points were surveyed as GCPs coordinates by total station Leica TCR1103. After the frames were processed, the final orientated models had a CPs accuracy up to less than a centimeter, so useful for a detailed architectural representation.

The integration of the laser scanning and photogrammetric clouds was used to build the mix of the facade's actual form. In order to create the polygonal model, the Geomagic wrap software was used to work on, as it offers, among other possibilities, the meshing of cloud models and inverse engineering tools from mesh to nurbs surfaces.

\section{SURVEYNG THE WOODEN MAQUETTE}

Domenico Rizzi's wooden model, kept safe at the Correr Museum in Venice, can be found inside a case closed by polycarbonate panels, which were momentarily removed for the purpose of the survey (figure 4).

The photogrammetric shots were taken in the first day with a Canon EOS 550D digital camera, equipped with an 18-135 lens, and mounted on a tripod. The parameters were set to: ISO 100, $18 \mathrm{~mm}$ focal length, 1 second exposure time, $\mathrm{f} / 3.5$ aperture. The long exposure time was necessary due to the room's lighting. The orientation of the 169 shots, taken with the Agisoft software again, allowed us to obtain a point cloud and the mesh needed to compare the data of the actual form and move on to the following reconstruction phase.

The second day was dedicated to laser scanning surveying, by using the Minolta 3D digitizer Vivid 9i triangulator, used for small and medium sized objects, equipped with 3 fixed focal length exchangeable lenses, differing on the needed detail precision. The medium lens was chosen to scan the $\mathrm{Ca}$ ' Venier of Lion's maquette, at a distance of about one meter for a total of 32 scans. A first alignment was done on site with the Polygon Editing Tool software (Konika Minolta) to verify the acquired data and export it to a polygonal mesh format.

Since the two sets of points clouds had about the same average density (up to one point every $2 \mathrm{~mm}$ on the measured object), the laser scanning data was integrated with those obtained photogrammetrically in the case of the wooden model as well, and processed in Geomagic to obtain a complete mesh.

\section{BUILDING THE UNFINISHED PALACE MODEL}

For the construction of the digital model of the hypothetic "finished" palace, the data of the wooden model was integrated with the actual form's.

The actual form's mesh was processed in Geomagic to obtain a polygonal model opportunely decimated with a definition of 500.00 triangles. Since the decimation involves losing information, an analysis of the different between the original point cloud and the "lightened" mesh, obtaining a standard deviation of $0.0191 \mathrm{~m}$, therefore with sufficient precision to virtually reproduce the façade. The accuracy of the digital model is related to the level of detail that should appear in the renderings but also to the detail that has to be obtained in $3 \mathrm{~d}$ printing later. From the two polygonal models we then moved to the construction of the final model in Blender $3 \mathrm{~d}$.

The model of the actual form was reprocessed through an operation of mesh tracing (re-topology) to obtain a complete $3 \mathrm{~d}$ surface in all its parts.

The maquette's mesh was orientated and scaled on the actual form's model, to complete the modeling of the entire prospect, once again through a re-topology of the detected surface (figure 5-6).

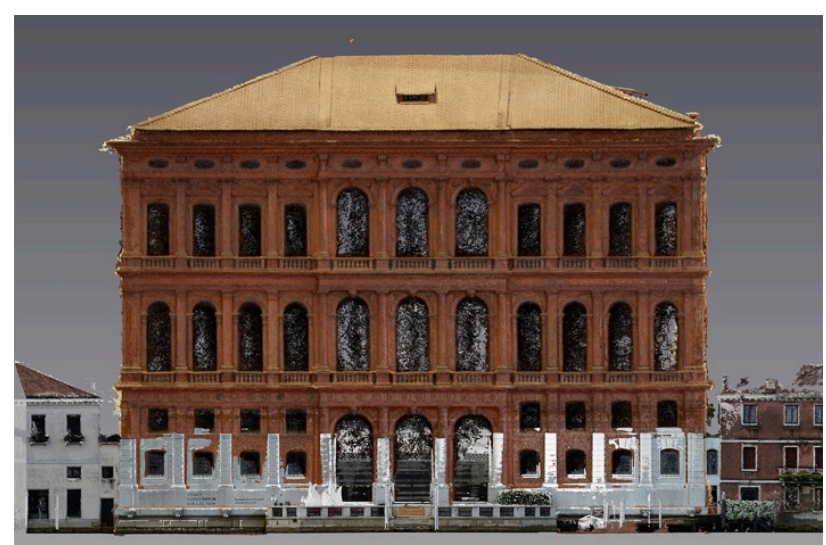

Figure 5. Comparison between the two datasets

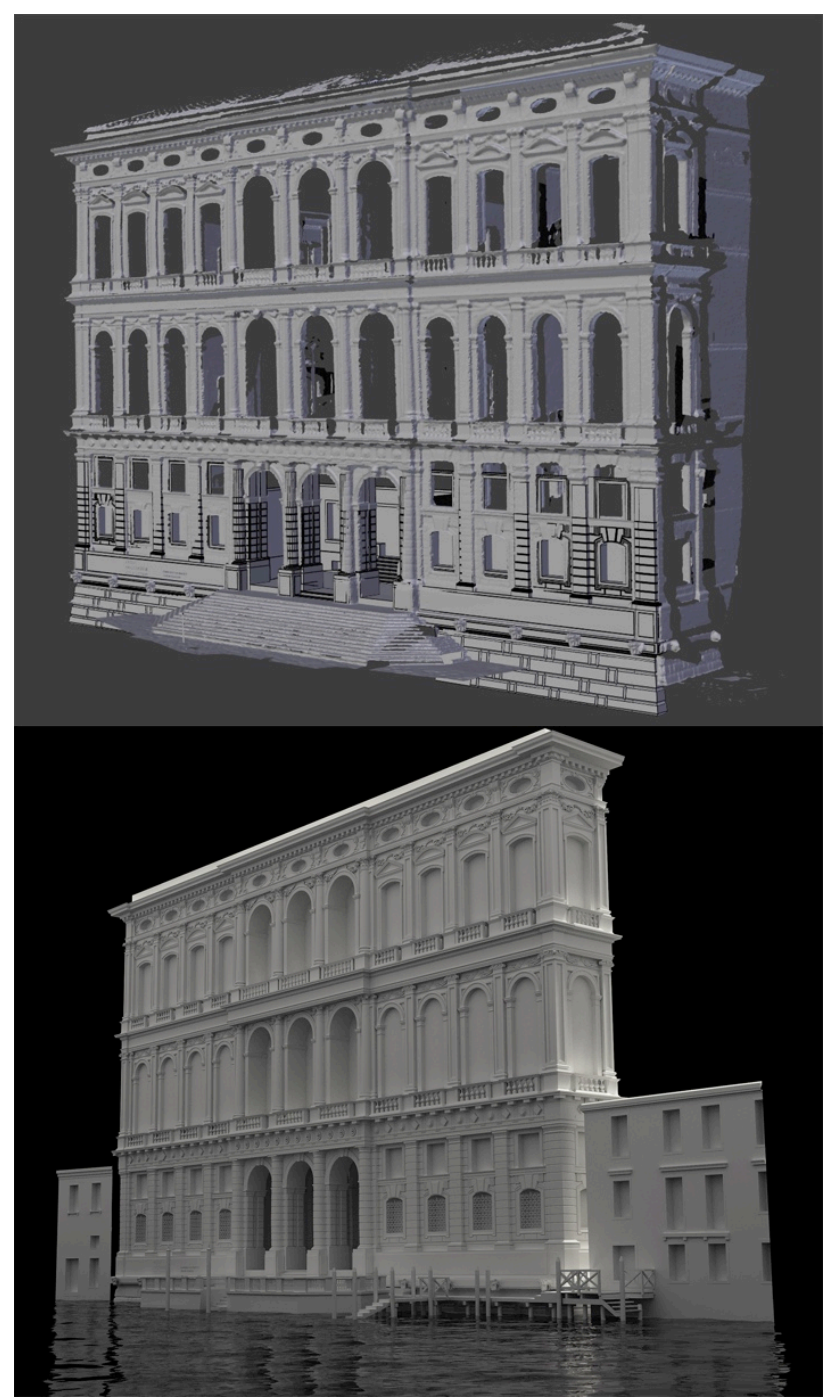

Figure 6. Creation of the prospect's model

\subsection{Computer graphics: from modeling to rendering and} animations

As for the photorealistic rendering we wished to obtain to verify the impact the Palace could have had, had it been finished, on the actual skyline of the Great Channel, the hybrid rendering engine (Unbiased+Biased) Cycles was used, integrated within Blender. 
The model was completed in this computer graphics phase, with the creation of the lateral prospects, of the covering pitches and the building's nearby.

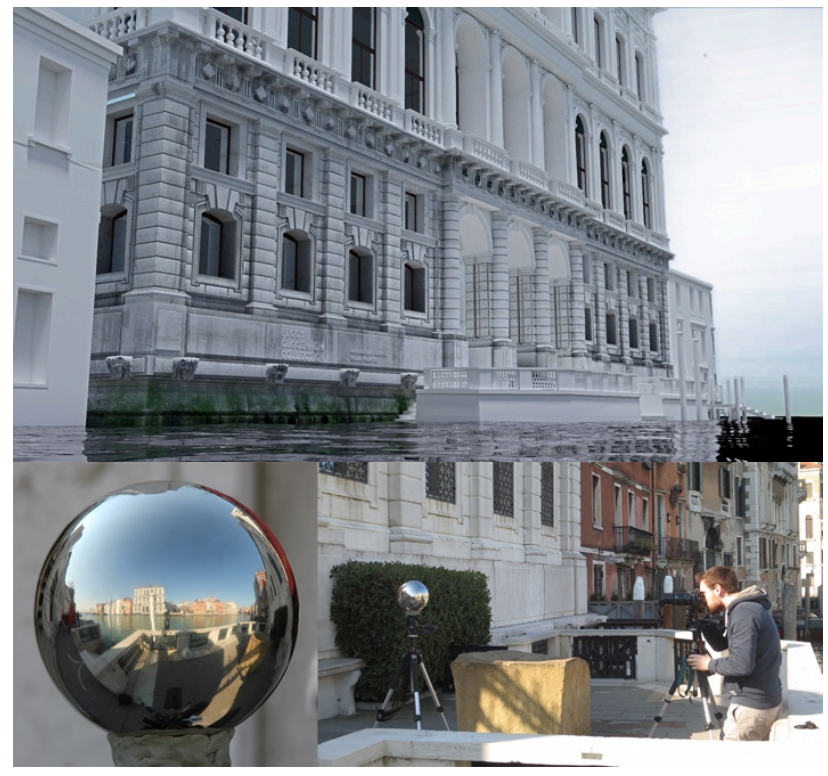

Figure 7. Phases of the photorealistic rendering with texture painting and HDRI technique

Particular attention was given to materials and the operation of texturing and unwrapping (the arrangement of the tridimensional polygons on the 2D space) of the meshes' texture. After creating a direct correspondence between 3D and $2 \mathrm{D}$ space, the local management of the texture on a $3 \mathrm{D}$ model was started.

Different levels of brushing were used, starting from the painting of the base texture on the 3D model and going on with brush strokes created by alpha channels to recreate the typical degradation of these types of buildings. In some materials, a layer using the vertex paint channel of the mesh was used, useful to locally regulate the gamma value of the texture, multiplied by the intensity value set at the vertexes. No bump and displacement channels were used, as the representation scale did not make it necessary (figure 7).

The illumination model was acquired with the "mirrorball" technique: the acquisition of the images happened on site with a reflective sphere mounted on a tripod and allowed for the surrounding environment to be captured with different exposure values, to compose a High Dynamic Range Image, used as environmental light in the tridimensional space, and Sun illumination was added to obtain shadows (environmental illumination, not being a vector, cannot create clear shadows).

The point of views were chosen in order for them to be inserted in the original context, so real and accessible point of views were selected. Thanks to the detected data, these points were identified in the 3D space of the Great Channel, obtaining a perfect alignment of the pictures taken and the rendering.

The post processing of the pictures allowed for the rendering to be integrated (purposefully broken down into render layers), as well as the photos for a photorealistic effect of the virtual building, inserted in the real context (figure 8).

The obtained results are particularly interesting, not only from the point of view of the rigorous process adopted to virtually conclude the building, but also the highly mimetic effect reached during the photorealistic renderings. The model, once inserted in the Venetian real context, provides an excellent example of virtual reconstruction in order to communicate both the history of $\mathrm{Ca}^{\prime}$ Venier dei Leoni and its potential impact in the Venetian skyline if it was been realized.

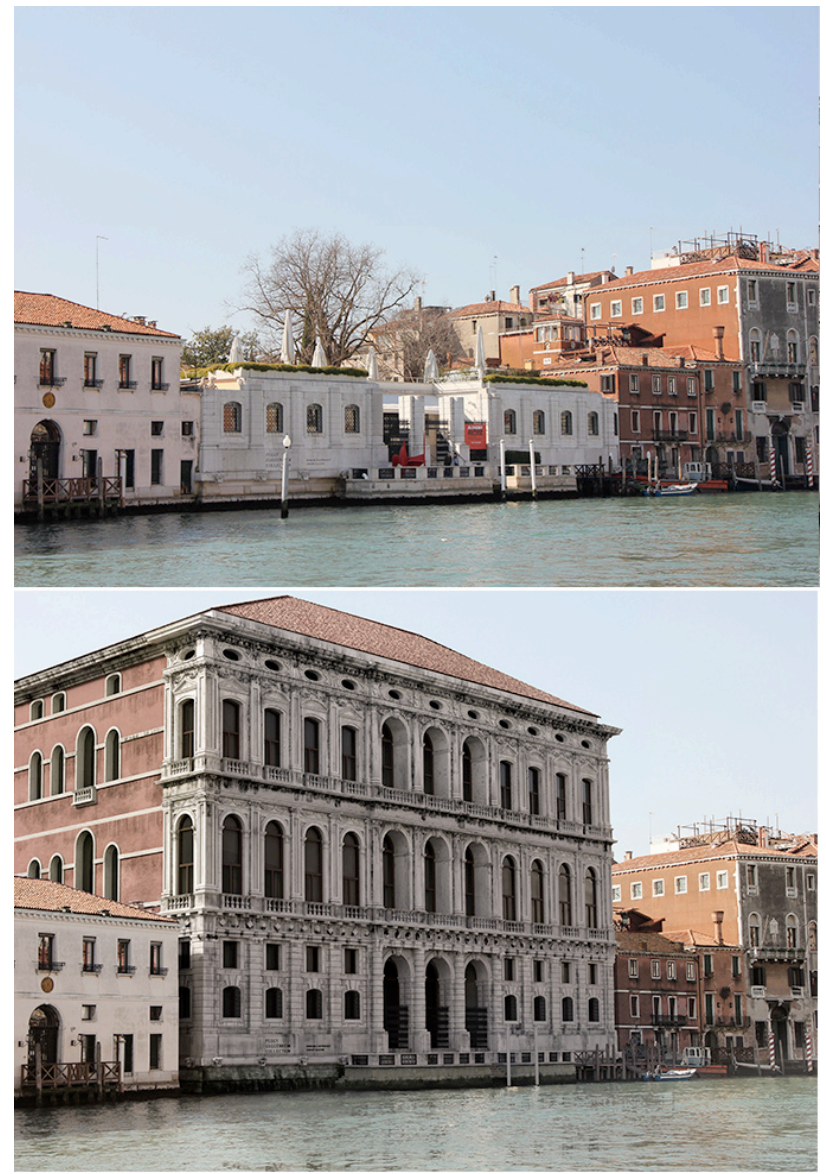

Figure 8. Comparison between the real view of the palace from the Great Channel and the hypothetic recreation of the complete façade

\section{3D PRINTING}

Besides the views and the animations, the project went on with the creation of a prototyped physical model (3d printing) of the palace which took the interpretation gained from the comparison of the actual form and the wooden model, into consideration.

To carry out the 3D print of a digital model, a series of factors must be taken into account, dictated by the technology that will be used: stereolitography, sintering, FDM, etc.

The technology used for the prototype of the $\mathrm{Ca}$ ' Venier of Lions was the laser sintering of polyamide powder: the geometrical information of the digital model is processed by the $3 \mathrm{D}$ printer's software by creating a series of images, then projected continuously allowing for the creation of the physical model (figura 9).

Operatively, the printing process begins with the drawing of a subtle powder layer, reinforced where necessary by laser sintering. Next the printing plate lowers (by $0,1 \mathrm{~mm}$ ) and this is repeated until it creates the entire object. The powder that is not consolidated remains to support the following layers.

An EOS P39S was used to print, with a print volume of $32 \times 32 \times 60 \mathrm{~cm}$ : the decision to obtain a model on a $1: 50$ scale entails a breakdown of the façade into the main bodies that make it up, obtaining three macro elements. This way, all the parts inside were able to be inserted in one print volume. 
The completion time amounted to 72 hours. The printed pieces were later put together in a single model of the façade (figure 10a-b).

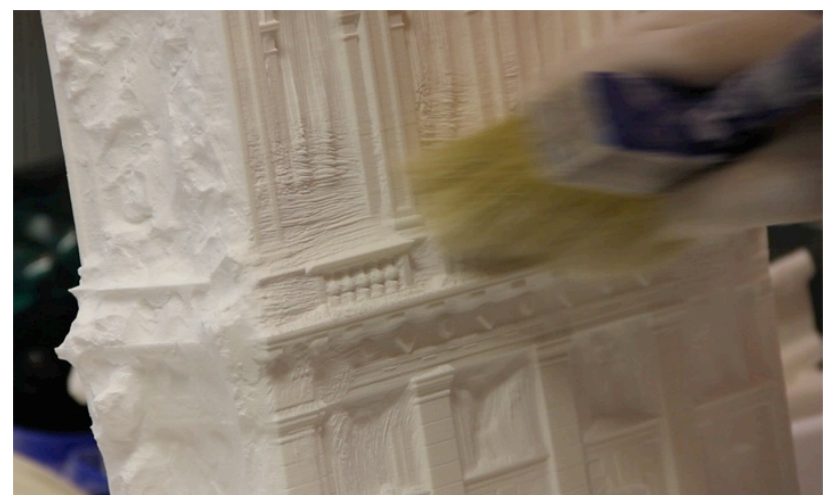

Figure 9. Cleaning the printed model's parts

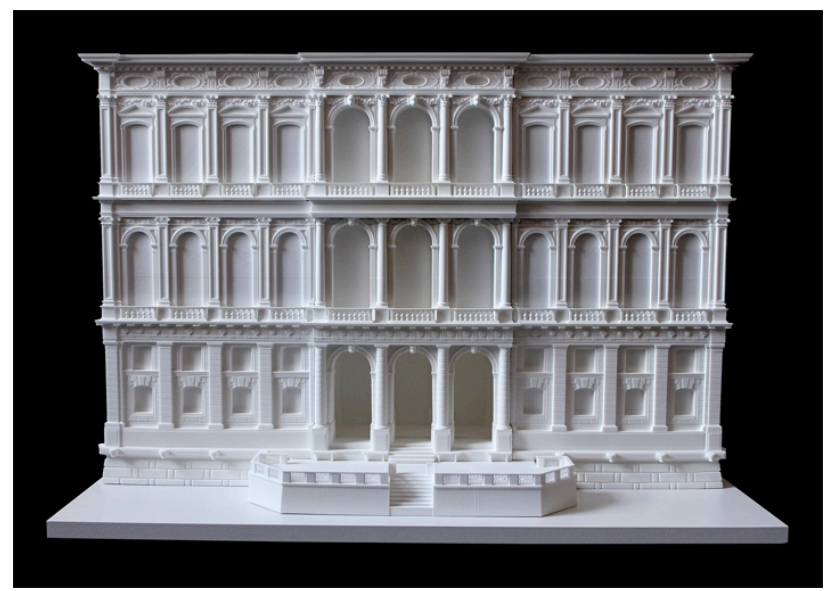

Figure 10. The prototyped model of the façade

\section{CONCLUSIONS}

The experience of the $\mathrm{Ca}$ ' Venier dei Leoni presented in this document highlights how geomatics, when applied to cultural goods, enhanced by equipment from different disciplines and fields of use, can provide the necessary applications to integrate the information on the city's history and architecture into a coherent whole entity, even when deriving from different sources, often not connected to each other. These processes are however closely linked thanks to the transversal contribution technologies give us: the diachronic process of $3 \mathrm{D}$ modeling for the recreation of lost or partially lost monuments should be developed through the integration and interpretation of historical and real data.

The multi-sensor approach was selected to fulfill measurements in order to integrate and compare geometric resolutions, which varies according to the types of objects (the real palace and the maquette).

It's important to consider which are the difficulties in acquisition in the Venetian urban context: the palace looks onto the Grand Canal, so its location puts some limits in in the acquisitions network. Laser-scanning and photogrammetry data integration is necessary and essential, but it has to be controlled by a topographic survey.

In this case study, the multi-sensor and multi-resolution data fusion was particularly interesting in the process of integration/ comparison of the real palace geometry and its wooden model: the different accuracies of sensors and platforms give an explanation to the change of scale between the two objects and make their models comparable.

The acquisition, modeling and $3 \mathrm{D}$ printing techniques represent a realistic kind of help for the conservation of cultural heritage, as they allow generating a digital copy of a real object. Also, the possibility to modify digital data does not only allow us to describe and explore, but also to compare information, considerably increasing the knowledge and valorization of a monument that is not visible anymore.

One more considerations has to be done on $3 \mathrm{~d}$ fabrication: as stated by Scopigno et al. (2015), even though 3D printing technologies still suffers of several restrictions, their use shows an interesting potential for future research on geometry processing, motivated by the specific needs of Cultural Heritage applications.

\section{REFERENCES}

Andrews, D. P., Bedford, J., \& Bryan, P. G., 2013. A Comparison of Laser Scanning and Structure from Motion as Applied to the Great Barn at Harmondsworth, UK. International Archives of the Photogrammetry, Remote Sensing and Spatial Information Sciences, Vol. XL(5/W2), pp. 31-36

Balletti, C., Berto, M., Gottardi, C., Guerra, F., 2014. 3D Technologies for the Digital Documentation of an Ancient Wooden Structure. International Journal of Heritage in the Digital Era (3)1: 19-32. doi :10.1260/2047-4970.3.1.19

Balletti, C., Brussa, N., Gottardi, C., Guerra, F., 2014. The documentation and reintegration of a lost past. ISPRS Ann. Photogramm. Remote Sens. Spatial Inf. Sci., II-5:49-55. doi:10.5194/isprsannals-II-5-49-2014, 2014.

Balletti, C., Guerra, F., 2015. The survey of cultural heritage: a long story. Rendiconti Lincei, 26: 115-125. doi:10.1007/s12210015-0411-8

Bastonero, P., Donadio, E., Chiabrando, F., and Spanò, A., 2014. Fusion of 3D models derived from TLS and image-based techniques for $\mathrm{CH}$ enhanced documentation, ISPRS Ann. Photogramm. Remote Sens. Spatial Inf. Sci., II-5, 73-80, doi:10.5194/isprsannals-II-5-73-2014.

Bitelli, G., Mannina, M., 2010. Geomatica e beni culturali: tecniche integrate per la documentazione e per la gestione dei beni culturali. Proceeding $14^{\circ}$ Conferenza Nazionale ASITA, Brescia, 2010. pp. 279-283

Bonfanti, C., Chiabrando, F., Rinaudo, F., 2013. TLS data for 2D representation and 3D modelling. Different approches tested in the case of san Giovanni in Salluzzo (CN) Italy. ISPRS Ann. Photogramm. Remote Sens. Spatial Inf. Sci., II-5/W1:37-42. doi:10.5194/isprsannals-II-5-W1-37-2013.

Bonora, V., Tucci, G., Vaccaro, V., 2005. 3D data fusion and multi-resolution approach for a new survey aimed to a complete model of Rucellai's chapel by Leon Battista Alberti in Florence. CIPA 2005 XX International Symposium, Torino, Italy, Vol. XXXVI, Part 5/C34, pp. 679-684

Camponogara, R, 2015. Palazzo Venier dei Leoni, metodi digitali per la costruzione del progetto non realizzato. Graduation Thesis, Università IUAV di Venezia.

Chiabrando, F., Lingua, A., Noardo, F., Spano, A., 2014. 3D modelling of trompe l'oeil decorated vaults using dense matching techniques, ISPRS Ann. Photogramm. Remote Sens. 
Spatial Inf. Sci., II-5: 97-104. doi:10.5194/isprsannals-II-5-972014.

Cignoni, P., Scopigno, R., 2008. Sampled 3d models for CH applications: a viable and enabling new medium or just a technological exercise. J. Comput. Cult. Herit. 1, 1, Article 2 (June 2008), 23 pages. doi:10.1145/1367080.1367082

Citlalli Gamez, S., Pillay, R., Tremeau, A., 2015. Data fusion of objects using techniques such as Laser Scanning, Structured Light and Photogrammetry for Cultural Heritage Applications. CCIW 2015, Lecture Notes in Computer Science, 9016: 208-224. doi:10.1007/978-3-319-15979-9 20

Guidi, G., Beraldin, J.A., Atzeni, C., 2004. High accuracy 3d modelling of cultural heritage: the digitizing of Donatello. IEEE Trans Image Process 13(3):370-380

Guidi, G., Remondino, F., Russo, M., Menna, F., Rizzi, A., Ercoli, S.2009. A multi-resolution methodology for the 3D modeling of large and complex archaeological areas. International Journal of Architectural Computing, 7(1):40-55

Remondino, F., Rizzi, A., 2010. Reality-based 3d documentation of natural and cultural heritage sites techniques, problems and examples. Applied Geomatics, Vol.2(3):85-100

Rinaudo, F., 2011. Il laser scanning terrestre per il rilievo e 1'analisi di Beni Architettonici e Archeologici. Proceeding $15^{\circ}$ Conferenza Nazionale ASITA, Reggia di Colorno, 2011. pp 1845-1846
Scopigno, R., Cignoni, P., Pietroni, N., Callieri, M. Dallepiane, M., 2014. Digital fabrication technologies fo Cultural Heritage (STAR). 12th Eurographics Workshops on Graphics and Cultural Heritage (EG GCH 2014), pp. 75-85

Stanco, F., Battiato, S., Gallo, G., 2011. Digital Imaging for Cultural Heritage Preservation. Taylor \& Francis Group.

Tucci, G., Bonora, V., Guardini, N., 2013. Analyses of the factors affecting 3D models resolution - application to the recording of vaults in Sangallo's house. ISPRS Ann. Photogramm. Remote Sens. Spatial Inf. Sci., II-5/W1:307-312 .doi:10.5194/isprsannals-II-5-W1-307-2013

Tcci, G., Bonora, V., 2015. Geomatics and management of atrisk cultural heritage. Rendiconti Lincei, 26: 105-114. doi:10.1007/s12210-015-0427-0

Wenzel, K., Abdel-Wahab, M., Cefalu, A., and Fritsch, D., 2012. High-Resolution Surface Reconstruction from Imagery for Close Range Culture Heritage Applications. Int. Arch. Photogramm. Remote Sens. Spatial Inf. Sci., XXXIX-B5, 133138, doi:10.5194/isprsarchives-XXXIX-B5-133-2012, 2012

\section{Sites:}

Peggy Guggenheim Collection, http://www.guggenheimvenice.it/inglese/museum/palazzo.html

PhotoScan Agisoft, http://www.agisoft.ru

Blender, http://www.blender.org/ 